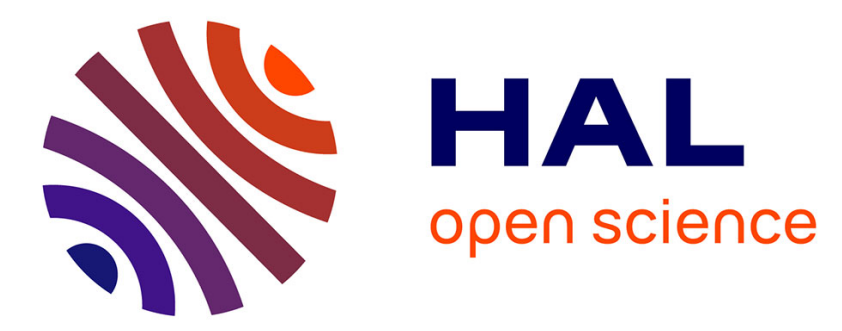

\title{
Ideal radiation source for plasma spectroscopy generated by laser ablation
}

\author{
Jorg Hermann, David Grojo, Emanuel Axente, Christoph Gerhard, Miloš \\ Burger, Valentin Craciun
}

\section{- To cite this version:}

Jorg Hermann, David Grojo, Emanuel Axente, Christoph Gerhard, Miloš Burger, et al.. Ideal radiation source for plasma spectroscopy generated by laser ablation. Physical Review E , 2017, 96 (5), pp.53210 - 53210. 10.1103/PhysRevE.96.053210 . hal-01655258

\section{HAL Id: hal-01655258 https://hal.science/hal-01655258}

Submitted on 4 Dec 2017

HAL is a multi-disciplinary open access archive for the deposit and dissemination of scientific research documents, whether they are published or not. The documents may come from teaching and research institutions in France or abroad, or from public or private research centers.
L'archive ouverte pluridisciplinaire HAL, est destinée au dépôt et à la diffusion de documents scientifiques de niveau recherche, publiés ou non, émanant des établissements d'enseignement et de recherche français ou étrangers, des laboratoires publics ou privés. 


\title{
Ideal radiation source for plasma spectroscopy generated by laser ablation
}

\author{
Jörg Hermann, ${ }^{1,}{ }^{*}$ David Grojo, ${ }^{1}$ Emanuel Axente, ${ }^{2}$ Christoph Gerhard, ${ }^{3}$ Miloš Burger, ${ }^{4}$ and Valentin Craciun ${ }^{2}$ \\ ${ }^{1}$ Aix-Marseille University, CNRS, LP3, 13009 Marseille, France \\ ${ }^{2}$ National Institute for Lasers, Plasma and Radiation Physics, 077125 Măgurele, Romania \\ ${ }^{3}$ Technical University of Applied Sciences Wildau, 15745 Wildau, Germany \\ ${ }^{4}$ University of Belgrade, Faculty of Physics, 11000 Belgrade, Serbia
}

(Received 24 July 2017; revised manuscript received 16 November 2017; published 28 November 2017)

\begin{abstract}
Laboratory plasmas inherently exhibit temperature and density gradients leading to complex investigations. We show that plasmas generated by laser ablation can constitute a robust exception to this. Supported by emission features not observed with other sources, we achieve plasmas of various compositions which are both uniform and in local thermodynamic equilibrium. These properties characterize an ideal radiation source opening multiple perspectives in plasma spectroscopy. The finding also constitutes a breakthrough in the analytical field as fast analyses of complex materials become possible.
\end{abstract}

DOI: 10.1103/PhysRevE.96.053210

\section{INTRODUCTION}

The search for an ideal radiation source for plasma spectroscopy has been one of the main challenges since the beginning of the development of plasma technologies $[1,2]$. Mostly motivated by the understanding of astrophysical plasmas, the early laboratory experiments were devoted to the generation of small-size plasmas to enable studies of atomic structures and the fundamentals of ionized diluted media [3]. Atmospheric plasmas such as arcs [1], shock tubes [4], and spark discharges [5] attracted attention due to their associated high collision rates that favor the establishment of equilibrium and facilitate the diagnostics in this way. With the introduction of the local thermodynamic equilibrium (LTE) concept, simplified theoretical descriptions of those plasmas became accessible through the statistical laws of equilibrium [6].

However, high-temperature laboratory plasmas are transitory by nature and particular efforts have been dedicated to the development of quasistationary plasma sources, for which the relaxation is slow enough to ensure LTE [7]. In turn, because the plasma lifetime becomes comparable to the characteristic times of heat and particle diffusion, these radiation sources are spatially nonuniform [8]. This translated the difficulties to another aspect, namely, the requirement of space-resolved observations and complex data analysis for accurate spectroscopic measurements [9].

Laser-generated plasma sources are relatively new. They have attracted strongly growing interest in the last two decades due to promising applications such as material analysis [10,11], material processing [12], and the generation of XUV ultrafast radiations [13]. Advances in highpower compact lasers today routinely enable the generation of highly reproducible plasmas, and spectroscopic measurements with intensified charge-coupled device detectors provide time-resolved plasma diagnostics with nanosecond resolution. Consequently, the understanding of plasmas produced by laser ablation has been greatly improved in recent years $[14,15]$.

*hermann@1p3.univ-mrs.fr
Compared to other atmospheric plasma sources, laser ablation plasmas are characterized by a much larger density (near solid density) during the early stage of expansion. This provides two improvements in the quest for an ideal uniform equilibrium source: (i) faster thermalization and (ii) slower diffusion processes. These are already exploited in material analysis with the recent advent of calibrationfree laser-induced breakdown spectroscopy $[16,17]$. However, the conditions of a uniform plasma remain only partially fulfilled because most studies are so far carried out in ambient air.

In this paper, we show that the plume generated in an inert background gas, such as argon, by ablation with ultraviolet laser pulses of nanosecond duration exhibits all the spectroscopic characteristics of a perfectly uniform plasma and is accurately described by the LTE model. We are not the first to find benefits of plasma generation in inert gases. However, the advantages reported so far were limited to an increased brightness, larger values of electron density and temperature, and a longer plasma lifetime [18,19]. For instance, in so-called tamped ablation experiments, high-pressure inert gas confines the vaporized material, and the laser energy is deposited in a small volume for optimized heating of high-temperature plasmas [20]. Here, we establish a different benefit with the formation of an isolating layer at the border of moderate temperature plasmas. Due to the high energy of the first excited state, the plasma electrons mostly interact with the inert gas atoms via elastic collisions, conserving thus their energy. This suppresses the spectroscopic signature of the cold border layer of the ablated vapor plasmas formed in air.

The unique properties of the laser-produced plasma are demonstrated by observing in its emission spectrum three independent features which are not found together for other laboratory plasmas: (i) the saturation of multiple transitions at the blackbody spectral radiance, (ii) the symmetric shape of strongly Stark-shifted lines, and (iii) the absence of absorption dips in highly self-absorbed resonance lines. To illustrate that the unique plasma properties are independent of its elemental composition, we report these emission features for ablation of very different target materials (steel, glass, pure indium). 


\section{EXPERIMENT}

The materials were irradiated with Nd:YAG laser pulses of a few $\mathrm{mJ}$ energy and a duration of several nanoseconds. The laser was operated in the ultraviolet spectral range to ensure efficient energy coupling to all sample materials. After beam focusing to a spot of $100 \mu \mathrm{m}$ diameter, a laser fluence of about $100 \mathrm{~J} \mathrm{~cm}^{-2}$ was incident on the surface of the samples. These were placed on a motorized sample holder in a vacuum chamber. During the experiments, the chamber was filled with argon at $5 \times 10^{4} \mathrm{~Pa}$ pressure or air at atmospheric pressure.

Optical emission spectroscopic measurements were performed by imaging the plasma with two lenses of 150 and $35 \mathrm{~mm}$ focal lengths onto the entrance of an optical fiber of $600 \mu \mathrm{m}$ core diameter. According to the image magnification $1: 5$, the entire plasma volume was observed. The fiber was coupled to the entrance of an echelle spectrometer with a resolving power of $9 \times 10^{3}$. Photon detection was ensured using an intensified charge-coupled device matrix detector. The spectral resolution and the apparatus response function were measured with appropriate calibrated lamps.

The spectra were recorded with a gate width $\Delta t_{g}$ short enough to make sure that the variations of electron density and plasma temperature during the recording were small compared to their absolute values. Thus, the recordings for $t=230,300$,
500, and $1250 \mathrm{~ns}$ were obtained with $\Delta t_{g}=50,100,200$, and $500 \mathrm{~ns}$, respectively. To enhance the signal-to-noise ratio, data acquisition was performed by averaging over multiple ablation events.

\section{OBSERVED EMISSION FEATURES}

\section{A. Blackbody radiation limit}

One characteristic feature of the ideal radiation source is easily observable for the plasma produced by ablation of steel thanks to the combination of two attributes: (i) the major element iron has a very rich emission spectrum including numerous lines of large transition probability; (ii) reliable spectroscopic data available for most $\mathrm{Fe}$ and $\mathrm{Fe}^{+}$transitions enable accurate plasma diagnostics. Thus, the most intense iron transitions are shown to reach the blackbody spectral radiance that is plotted in Fig. 1 for the temperature deduced from the intensity distribution of optically thin $\mathrm{Fe}$ and $\mathrm{Fe}^{+}$ lines. Moreover, it is shown for three narrow spectral ranges (labeled A, B, and C) that the measured spectrum is accurately described by the spectral radiance of a spatially uniform plasma in local thermodynamic equilibrium.
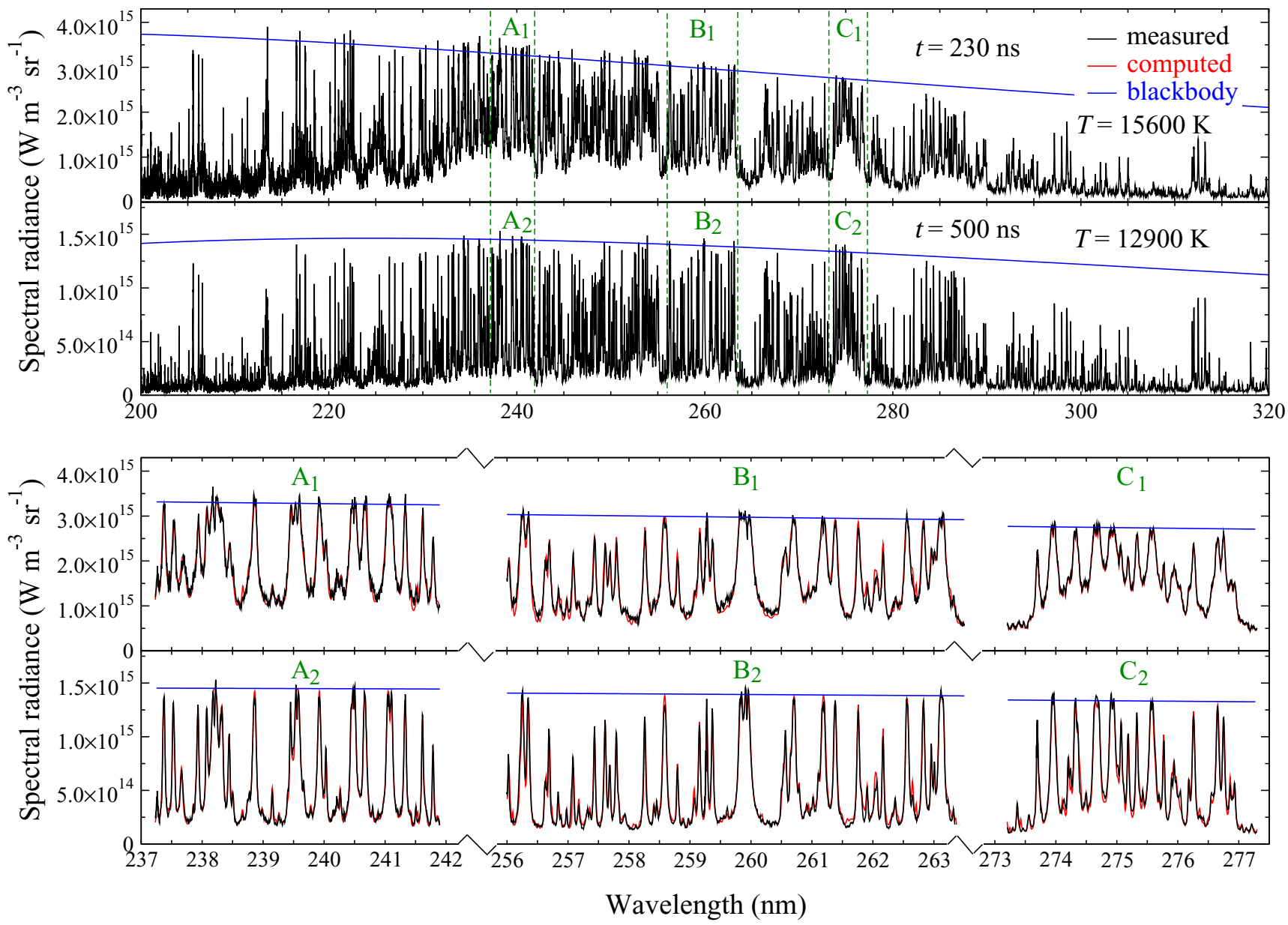

FIG. 1. Space-integrated spectra recorded during laser ablation of steel in argon at different times. The strongest transitions are shown to saturate at the blackbody spectral radiance. The temperature was deduced from the intensity distribution of optically thin lines. The excellent agreement with the spectral radiance computed using Eq. (1) shows that the emission originates from a uniform plasma in LTE. 


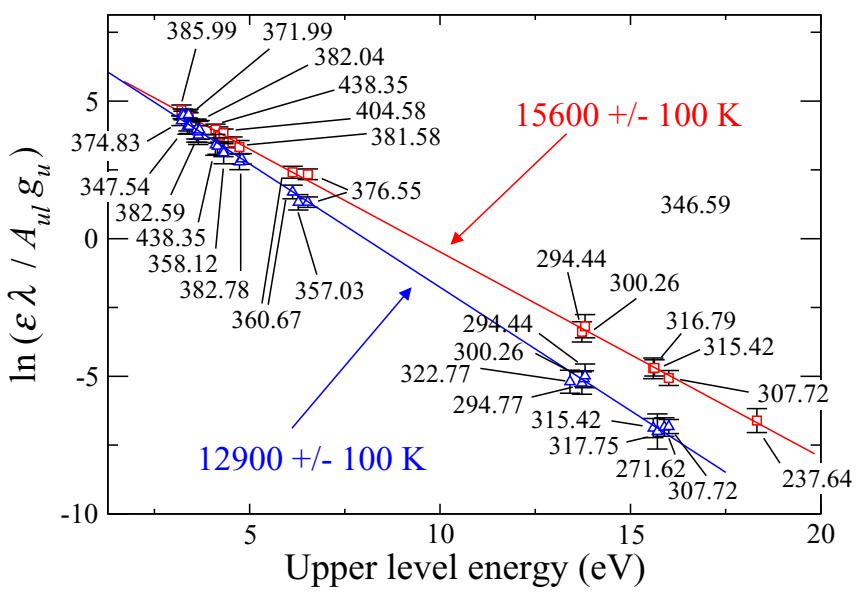

FIG. 2. Saha-Boltzmann plot of $\mathrm{Fe}$ and $\mathrm{Fe}^{+}$transitions for $t=$ $230 \mathrm{~ns}$ (squares) and $500 \mathrm{~ns}$ (triangles).

In practice, the spectral radiance of a rigorously uniform plasma in LTE is calculated by [21]

$$
B_{\lambda}=U_{\lambda}\left(1-e^{-\alpha(\lambda) L}\right),
$$

where $U_{\lambda}$ is the blackbody spectral radiance, $\alpha(\lambda)$ is the wavelength-dependent absorption coefficient, and $L$ is the plasma size along the line of sight. The optical thickness is given by $\tau=\int \alpha(z) d z=\alpha L$. In the optically thin case $(\tau \ll 1)$, Eq. (1) becomes

$$
B_{\lambda}=\varepsilon_{\lambda} L,
$$

where the emission coefficient $\varepsilon_{\lambda}$ is related to the absorption coefficient via Kirchhoff's law of thermal radiation, $\varepsilon_{\lambda} / \alpha=$ $U_{\lambda}$. In the opposite case of large optical thickness $(\tau \gg 1)$, Eq. (1) simplifies to

$$
B_{\lambda}=U_{\lambda} .
$$

The spectral radiance becomes independent of the plasma size and depends on temperature only.

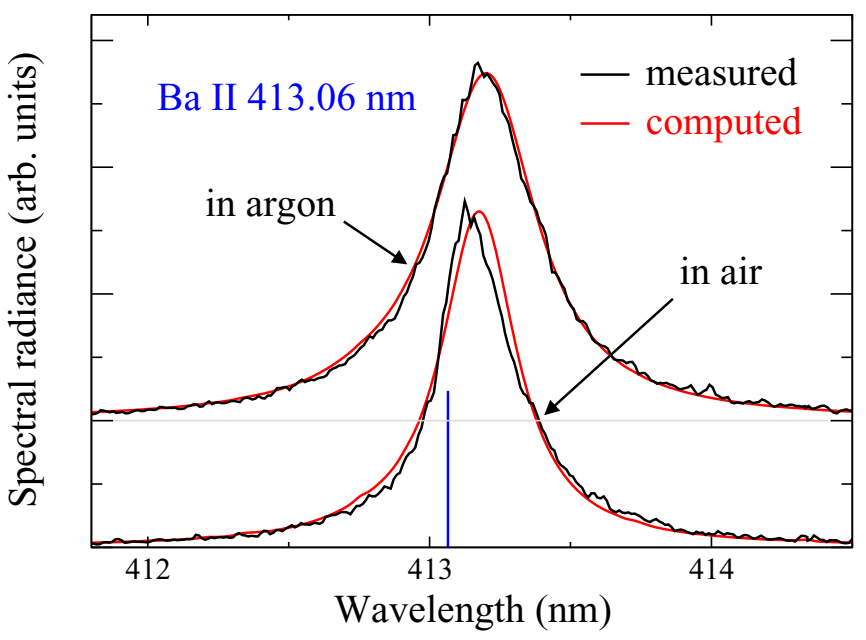

FIG. 3. Ba II $413.06 \mathrm{~nm}$ line profile recorded during laser ablation of barite crown glass for $t=500 \mathrm{~ns}$. Comparison to the spectral radiance computed for a uniform LTE plasma. The blue line indicates the resonance wavelength of the transition.
Considering all relevant transitions between bound and free excitation levels, the absorption coefficient writes

$$
\alpha(\lambda)=\sum_{i} \alpha_{\text {line }}^{(i)}(\lambda)+\alpha_{\text {ion }}(\lambda)+\alpha_{I B}(\lambda),
$$

where the sum includes the absorption coefficients $\alpha_{\text {line }}^{(i)}$ of transitions between bound levels. The terms $\alpha_{\text {ion }}(\lambda)$ and $\alpha_{I B}(\lambda)$ account for absorption through radiative ionization and inverse bremsstrahlung, respectively. The absorption coefficient of transitions between bound levels is given by [6]

$$
\alpha_{\text {line }}(\lambda)=\pi r_{0} \lambda^{2} f_{l u} n_{l} P(\lambda)\left(1-e^{-h c / \lambda k T}\right),
$$

where $r_{0}$ is the classical electron radius, $h$ is the Planck constant, $c$ is the vacuum light velocity, $T$ is the temperature, and $f_{l u}$ and $n_{l}$ are the absorption oscillator strength and the lower level population number density of the transition, respectively. The normalized line profile $P(\lambda)$ is calculated considering Doppler and Stark effects that are the dominant mechanisms of spectral line broadening in strongly ionized laser-produced plasmas [22]. The spectroscopic data were taken from NIST and Kurucz databases [23,24]. Most of the Stark broadening parameters were not found in the literature and therefore measured in a separate experiment, assuming a linear dependence of Stark widths and shifts on electron density $n_{e}$ [25]. For the calculation of the LTE plasma composition, we refer to previous work [26,27].

To compare the measured spectrum to the spectral radiance computed according to Eq. (1), $B_{\lambda}$ is convoluted with the apparatus spectral profile. Moreover, instead of calculating the absorption coefficients due to ionization and inverse bremsstrahlung [see Eq. (4)], the amount $\alpha_{\text {ion }}(\lambda)+\alpha_{I B}(\lambda)$ was adjusted so that the intensity of computed continuum radiation equals the measured continuum intensity.

The values of $T, n_{e}, L$ and the elemental composition were deduced by comparing the measured spectra to the computed spectral radiance [Eq. (1)], applying the iterative measurement algorithm presented previously [27]. Only isolated transitions of small optical thickness are considered to ensure accurate plasma diagnostics. The plasma size along the line of sight was deduced from the intensity ratio between optically thin and thick lines using Eqs. (2) and (3). We obtain $L=0.4$ and $0.5 \mathrm{~mm}$ for the spectra recorded at $t=230$ and $500 \mathrm{~ns}$, respectively. Once the plasma properties are known, the plasma emission spectrum is computed for the ranges of interest.

To point out the high accuracy of the present plasma diagnostics, the temperature measurement through the intensity ratio of spectral lines from atoms and ions is illustrated by the Saha-Boltzmann plot in Fig. 2. Here, the emission coefficient of each line is deduced from the measured spectrum using $\varepsilon=$ $\varepsilon_{\text {comp }} B_{\text {meas }} / B_{\text {comp }}$, where $B_{\text {meas }}$ and $B_{\text {comp }}$ are the measured and computed line-integrated spectral radiances, respectively. The accurate description of spectral lines from both atoms and ions by a unique slope supports the hypothesis of a spatially uniform plasma. As a consequence of the variations of optical thickness and line interferences, the lines selected for $T$ measurements depend on the recording time. Typically, transitions of small Stark broadening parameter are used at early times, whereas strongly broadened lines are selected in priority at large delays. 


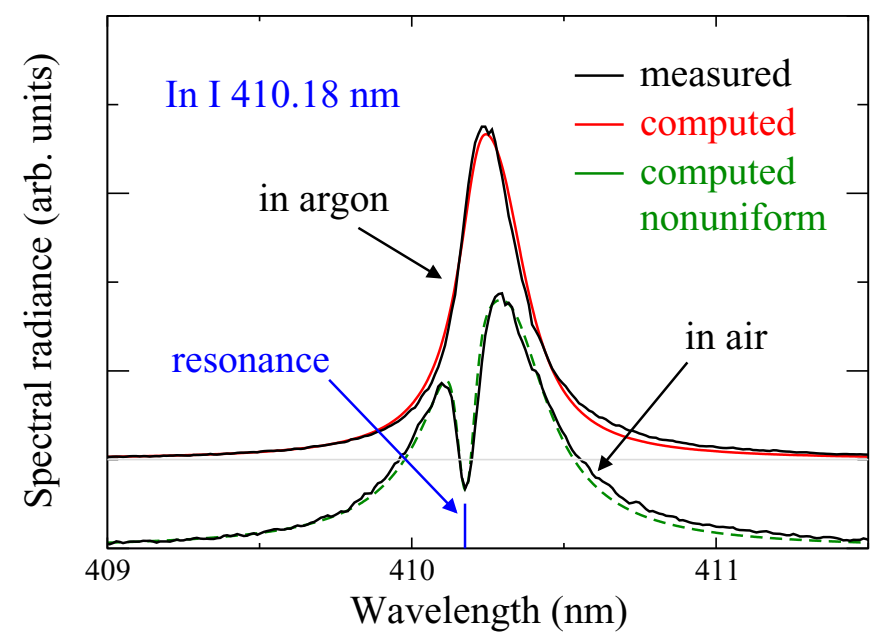

FIG. 4. In I $410.18 \mathrm{~nm}$ resonance line recorded during laser ablation of indium for $t=500 \mathrm{~ns}$. The measurements in argon and air are compared to the spectral radiances of a uniform and a nonuniform LTE plasma, respectively.

\section{B. Symmetry of Stark-shifted lines}

Another feature demonstrating the uniform character of the plasma is shown in Fig. 3 for laser ablation of barite crown glass, a material of properties and composition completely different from those of steel. The glass contains barium that exhibits strongly Stark-shifted lines in its emission spectrum. The spectral shape of such a line is displayed for irradiation in air and argon background gas. The asymmetric line profile in air is due to the contributions from volumes of different electron densities, illustrating thus the nonuniform $n_{e}$ distribution of the ablation plume produced in air. Contrarily, the symmetric profile in argon evidences the uniform character of the plasma produced in the inert gas.

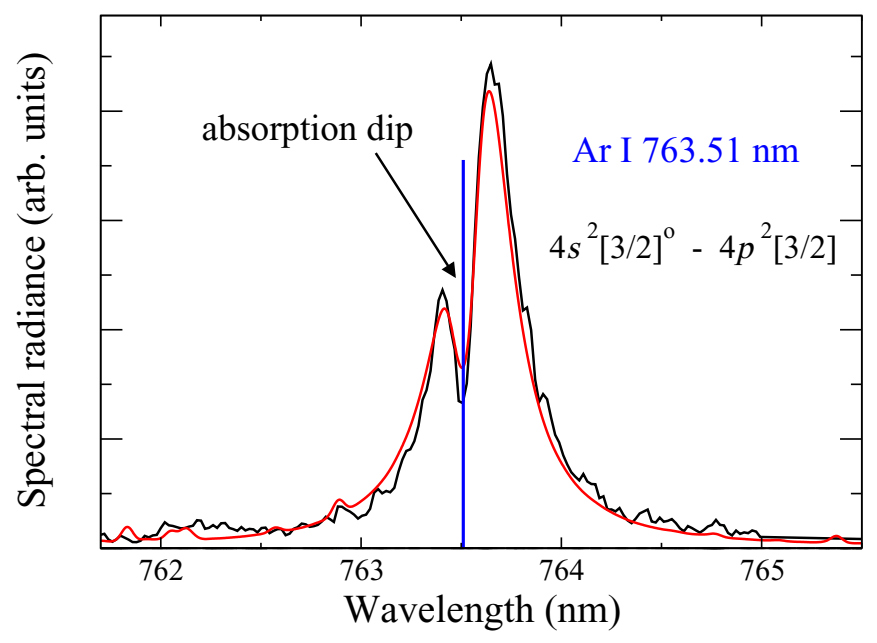

FIG. 5. Measured (black line) and computed (red line) shapes of an Ar transition toward the metastable state. The recording was performed during ablation of steel for $t=500 \mathrm{~ns}$. The simulation was obtained by adding two layers to the laser plasma, representing the heated gas at the vapor-gas contact front and the adjacent colder gas. The absorption dip was observed for each investigated material.

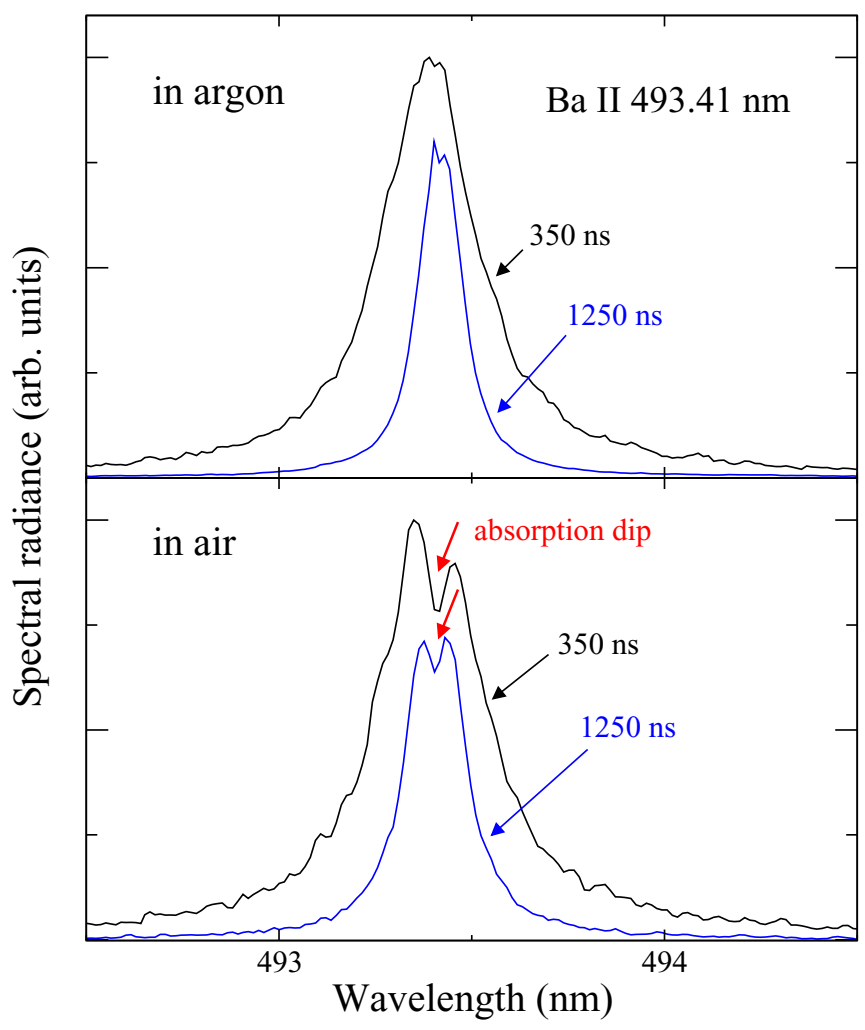

FIG. 6. Ba II $493.41 \mathrm{~nm}$ line profile recorded during laser ablation of barite crown glass in argon and air for different times.

\section{Absorption at the plasma border}

An even more direct demonstration of the uniform character of the plasma is given by the spectral shape of strongly self-absorbed resonance lines. Laboratory plasmas typically exhibit a cold border layer in which the ground state population number densities are particularly large. According to the moderate electron density in the border layer, absorption occurs exclusively in a narrow range close to the resonance wavelength. Hence, resonance lines typically exhibit an absorption dip, as shown in Fig. 4 for the In I $410.18 \mathrm{~nm}$ transition observed during laser ablation of indium in air. When ablation occurs in argon, the resonance line does not exhibit the absorption dip, proving thus that the cold border layer is not formed in the ablated vapor plasma. The presence or absence of the cold plasma border for ablation in air or argon, respectively, was evidenced for all kinds of investigated sample materials and for a large time range (see the Appendix).

The uniform spatial distribution of the ablation plume produced in argon is explained by the formation of an isolating layer at the vapor-gas contact front. Indeed, argon atoms are characterized by a large energy gap between the ground state and the first excited state $(11.55 \mathrm{eV})$. The rates of collisional excitation and deexcitation are therefore very small and the plasma electrons interact with argon atoms mostly through elastic collisions and conserve their energy.

Even if the major part of the plasma volume is characterized by uniform spatial distributions of temperature and density, gradients always exist at the border. In the present case, the expanding plume is in contact with the cold background gas that is heated by the shock wave and, on a longer time scale, 


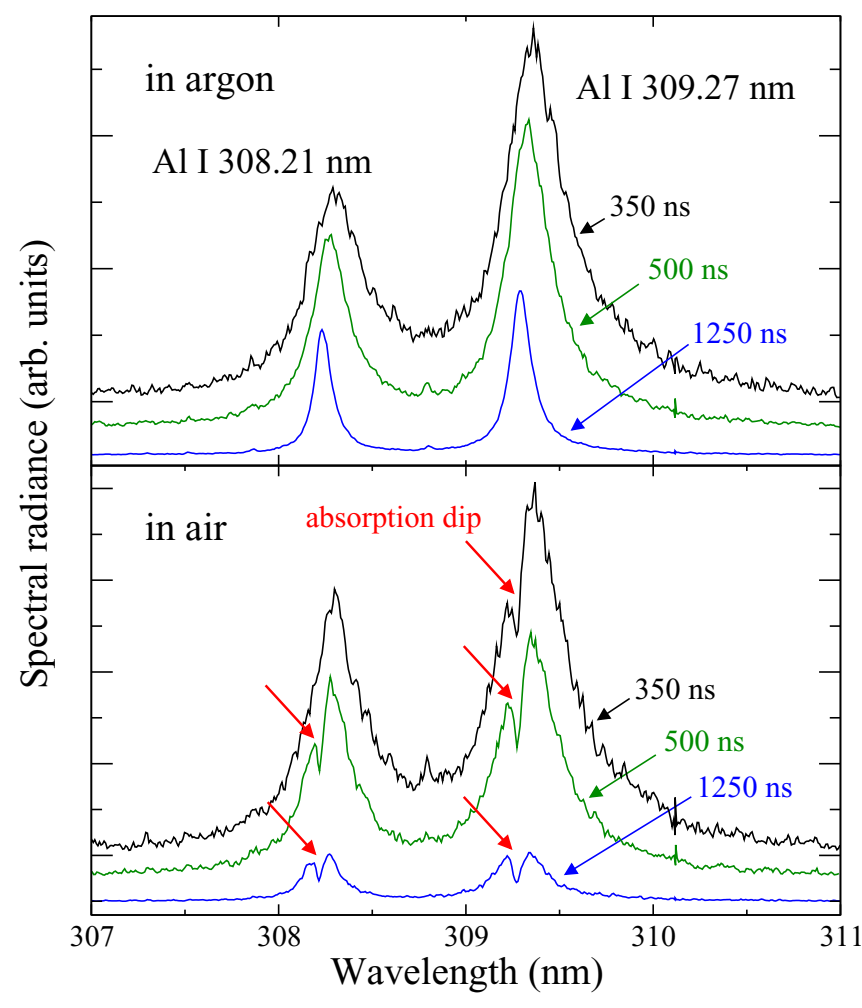

FIG. 7. Aluminum resonance lines recorded during laser ablation of an aluminum alloy in argon and air for different times.

by heat diffusion. The nature of the gas has a strong influence on the exchange of energy between the ablated vapor plume and the ambient gas. For ablation in argon, the gradients of temperature and density appear in the background gas, close to the vapor-gas contact front, as illustrated by the absorption dip of the argon transition displayed in Fig. 5. The Ar I $763.51 \mathrm{~nm}$ line decays towards the $4 s^{2}[3 / 2]^{\circ}$ metastable state that is the first excitation level of the argon atom. Separated by an energy of $11.55 \mathrm{eV}$ from the ground state, the collisional excitation to and deexcitation from the metastable state have low probabilities. The $4 s^{2}[3 / 2]^{\circ}$ state is therefore the bottleneck in the relaxation process of argon atoms and its population number density is expected to be overpopulated compared to the equilibrium value.

The disparity from the Boltzmann equilibrium distribution of the population number densities of argon atoms was evidenced through the calculation of the Ar I $763.51 \mathrm{~nm}$ line profile. In fact, according to the large excitation energy, the equilibrium population number density of the $4 s^{2}[3 / 2]^{\circ}$ state is far too small to cause significant absorption of Ar I $763.51 \mathrm{~nm}$. Thus, to simulate the absorption dip displayed in Fig. 5, the population number density of the metastable state was increased by more than eight orders of magnitude with respect to the equilibrium case.

\section{CONCLUSION}

We show by observation of three independent emission features that laser ablation under appropriate irradiation conditions generates a plasma of unique properties, characterized by spatially uniform distributions of temperature and densities and well described by the state of local thermodynamic equilibrium. The combination of both properties enables simple and robust modeling of the plasma emission spectrum with an excellent level of accuracy. Being achievable independently of the elemental composition, the unique properties qualify the laser-induced plasma as an ideal radiation source, opening multiple perspectives in plasma spectroscopy with straightforward access to fundamentals in plasma and atomic physics, and accurate measurements of spectroscopic data. Moreover, this constitutes a breakthrough in the analytical field as accurate elemental analyses of complex materials become possible without any requirement of calibration with standard samples. The present findings are thus foreseen to directly impact interdisciplinary challenges in various fields such as food security, environmental risks, and biomedicine [10].

\section{ACKNOWLEDGMENT}

The research leading to these results has received funding from LASERLAB-EUROPE (Grants Agreement No. 284464 and No. 654148, European Union's Horizon 2020 research and innovation programme).

\section{APPENDIX: ADDITIONAL EXAMPLES}

The absorptions dips in resonance lines are observed for laser ablation in air while they are not observable for ablation in argon. The feature is independent of the ablated sample material and can be observed at various times, as shown in Figs. 6 and 7. This shows that the cold plasma border within the ablated material vapor is formed in air at early time, while it is absent in argon within the entire time range of interest.
[1] A. G. Shenstone, Nature (London) 114, 934 (1924).

[2] L. S. Ornstein and T. Bouma, Phys. Rev. 36, 679 (1930).

[3] H. Alfvén, Astrophys. Space Sci. 54, 279 (1978).

[4] W. L. Wiese, H. F. Berg, and H. R. Griem, Phys. Rev. 120, 1079 (1960).

[5] C. A. Bye and A. Scheeline, Appl. Spectrosc. 47, 2022 (1993).

[6] H. R. Griem, Plasma Spectroscopy (Academic, New York, 1964).
[7] W. L. Wiese, Spectrochim. Acta Part B: Atom. Spectrosc. B 46, 831 (1991).

[8] T. Belmonte, C. Noël, T. Gries, J. Martin, and G. Henrion, Plasma Sources Sci. Technol. 24, 064003 (2015).

[9] R. Álvarez, A. Rodero, and M. C. Quintero, Spectrochim. Acta Part B: Atom. Spectrosc. 57, 1665 (2002).

[10] B. Kearton and Y. Mattley, Nat. Photon. 2, 537 (2008).

[11] S. M. McLennan, R. B. Anderson, J. F. Bell, J. C. Bridges, and M. S. Team, Science 343, 1244734 (2014). 
[12] I. W. Boyd and F. Micheli, Nature (London) 321, 649 (1986).

[13] M. Nishikino and T. Kawachi, Nat. Photon. 8, 352 (2014).

[14] G. Cristoforetti, A. D. Giacomo, M. Dell'Aglio, S. Legnaioli, E. Tognoni, V. Palleschi, and N. Omenetto, Spectrochim. Acta Part B: Atom. Spectrosc. 65, 86 (2010).

[15] D. W. Hahn and N. Omenetto, Appl. Spectrosc. 66, 347 (2012).

[16] E. Tognoni, G. Cristoforetti, S. Legnaioli, and V. Palleschi, Spectrochim. Acta Part B: Atom. Spectrosc. 65, 1 (2010).

[17] J. Hermann, A. Lorusso, A. Perrone, F. Strafella, C. Dutouquet, and B. Torralba, Phys. Rev. E 92, 053103 (2015).

[18] S. S. Harilal, C. V. Bindhu, V. P. N. Nampoori, and C. P. G. Vallabhan, Appl. Phys. Lett. 72, 167 (1998).

[19] A. J. Effenberger and J. R. Scott, Sensors 10, 4907 (2010).

[20] J. C. Fernández et al., Phys. Plasmas 13, 056319 (2006).

[21] J. Cooper, Rep. Prog. Phys. 29, 35 (1966).
[22] X. Z. Zhao, L. J. Shen, T. X. Lu, and K. Niemax, Appl. Phys. B: Photophys. Laser Chem. 55, 327 (1992).

[23] A. Kramida, Y. Ralchenko, and J. Reader, NIST Atomic Spectra Database (version 5.3) (National Institute of Standards and Technology, Gaithersburg, MD, 2015).

[24] P. L. Smith, C. Heise, J. R. Esmond, and R. L. Kurucz, Atomic spectral line database built from atomic data files from R. L. Kurucz CD-ROM 23, (2011) (unpublished).

[25] M. Burger and J. Hermann, Spectrochim. Acta Part B: Atom. Spectrosc. 122, 118 (2016).

[26] J. Hermann and C. Dutouquet, J. Appl. Phys. 91, 10188 (2002).

[27] C. Gerhard, J. Hermann, L. Mercadier, L. Loewenthal, E. Axente, C. R. Luculescu, T. Sarnet, M. Sentis, and W. Viöl, Spectrochim. Acta Part B: Atom. Spectrosc. 101, 32 (2014). 